\title{
Robust positively invariant sets for state dependent and scaled disturbances
}

\author{
Rainer M. Schaich ${ }^{\dagger}$ and Mark Cannon ${ }^{\dagger, \ddagger}$
}

\begin{abstract}
This paper introduces methods of deriving and computing maximal robust positively invariant sets for linear discrete time systems with additive model uncertainty. Two types of uncertainty are considered: state dependent uncertainty, which can handle multiplicative parametric model uncertainty as well as linearisation errors for nonlinear systems, and scaled sets of uncertainty. We provide a framework for analysing both types of uncertainty with illustrative examples.

Index Terms - constrained control, positively invariant sets, robust control
\end{abstract}

\section{INTRODUCTION}

The analytic properties of disturbance invariant sets have been widely studied (e.g. [1]) since their introduction in [2], [3]. A disturbance invariant set $\mathscr{X}$ is a subset of the state constraint set $\mathcal{X}_{0} \subseteq \mathbb{R}^{n}$ containing states $x$ of the perturbed linear system

$$
x^{+}=\Psi x+v
$$

(where $\Psi$ is Hurwitz, $x, x^{+} \in \mathcal{X}_{0}$, and $v \in \mathscr{V}$ ), such that the successor state $x^{+}$is contained in $\mathscr{X}$ for all possible realisations of the unknown disturbance $v$. Thus a disturbance invariant set is a type of robust positively invariant (RPI) set. This is summarised in the implicit definition

$$
\mathscr{X}=\{x: \Psi x+v \in \mathscr{X} \forall v \in \mathscr{V}\} .
$$

In many applications we are interested in the largest such set, denoted $\mathcal{X}^{\infty}$, which is given by the union of all RPI sets $\mathscr{X} \subseteq \mathcal{X}^{\infty}$. This set is known as the maximal robust positively invariant (MRPI) set and has been used extensively, for example, to define terminal regions in robust model predictive control [4]. Although analytical properties of these sets were derived at the time of their introduction, algorithms for numerically computing MRPI sets were introduced much later [5]-[7]. Earlier algorithms did not guarantee finite determinability of $\mathcal{X}^{\infty}$, see e.g. [8], or were not guaranteed to produce the maximal RPI set, e.g. [9]. However for the case of disturbances belonging to a fixed set $\mathscr{V}$, methods of computing $\mathcal{X}^{\infty}$ are now well established [1].

This paper considers sets of disturbances that depend on parameters. First we consider state-dependent sets with the irredundant representation

$$
\mathcal{V}(x)=\{v: G v \leq H(x)\},
$$

where $G$ is a real matrix, $H(x)$ is a convex piecewise affine function, and the inequality applies elementwise. For the case that $\mathscr{V}=\mathcal{V}(x)$ we provide conditions for convexity and finite determination of $\mathcal{X}^{\infty}$ and discuss computation of $\mathcal{X}^{\infty}$. The case of disturbances depending on states (or control

\footnotetext{
$\dagger$ Department of Engineering Science, University of Oxford, OX1 3PJ.

¥ Corresponding author, mark.cannon@eng.ox.ac.uk.
}

inputs) is considered in [10], [11] but the convexity analysis of this paper and the associated computational approach are novel. State dependent disturbance constraints can account for linearisation errors, as shown in section IV, as well as more general multiplicative parametric model uncertainty.

The second case considered is that of scaled disturbance sets of the form

$$
\mathcal{V}(\theta)=\{v: G v \leq(1+\theta) \mathbf{1}\},
$$

for scalar $\theta>-1$. For $\mathscr{V}=\mathcal{V}(\theta)$ we compute sets $\mathcal{Z}^{\infty}$ in $(x \times \theta)$-space with the property that the intersection, $\left.\mathcal{Z}^{\infty}\right|_{\hat{\theta}}$, with the subspace on which $\theta=\hat{\theta}$ for given $\hat{\theta}$ is an MRPI set. To our best knowledge, this setup has not been considered in the literature. This type of disturbance constraint can be used to study the sensitivity of the MRPI set to changes in the disturbance strength.

The structure of the paper is as follows: We introduce the notion of parametric convexity In Section II and discuss its implications, in particular as a condition for convexity of the MRPI set. The algorithm to compute the MRPI set for state dependent disturbance constraints and its finite determinability are discussed in Section III. Section IV illustrates the algorithm using a nonlinear model of a magnetically levitated ball. The case of scaled disturbance constraints is discussed in Section V, which gives the algorithm for computing the parametrised MRPI set and proves its finite determinability. Section VI illustrates the use of parametrised MRPI sets using the levitating ball example, and illustrates a robustness analysis of the parametrised MRPI sets with a numerical example. Section VII provides conclusions.

Throughout this paper we refer to sets that can be represented as intersections of finite numbers of half spaces as polyhedra, and to bounded polyhedra as polytopic sets. For sets $\mathcal{A}, \mathcal{B} \subset \mathbb{R}^{n}$, the Minkowski set addition is denoted $\mathcal{A} \oplus \mathcal{B}=\{c: c=a+b \forall a \in \mathcal{A}, b \in \mathcal{B}\}$. We denote the column vector of ones in appropriate dimensions as 1 , the logical AND operator as $\wedge$, and index sets as $\mathcal{I} \subset \mathbb{N}$.

\section{PARAMETRICALly CONVEX SET OPERATIONS}

This section discusses sets that depend on a parameter such as the state of (1) (so called point-to-set maps, see [12]), and extends the existing set algebra [1] to accommodate such sets. We present the general case first, then illustrate it with the example of a pointwise polyhedral set in the form of (3)

Definition 2.1 (Parametric Convexity): Let $X \subseteq \mathbb{R}^{n}, Y \subseteq$ $\mathbb{R}^{m}$, let $\mathcal{P}(Y)$ denote the power set of $Y$, and $T: X \rightarrow$ $\mathcal{P}(Y), X \ni s \mapsto T(s) \subset Y$ be a continuous point-to-set map. The map $T$ is called parametrically convex if it satisfies

$$
T\left(\lambda s_{1}+(1-\lambda) s_{2}\right) \subseteq \lambda T\left(s_{1}\right) \oplus(1-\lambda) T\left(s_{2}\right)
$$


for all $s_{1}, s_{2} \in X$ and $\lambda \in[0,1]$.

The difference of sets can be defined for parametrised sets analogously to the Pontryagin difference [7] as follows:

Definition 2.2 (Parametric Pontryagin Difference): Let $S \subseteq X$ and let $T: X \rightarrow \mathcal{P}(X)$ be a continuous point-to-set map, then the parametric Pontryagin difference $S \ominus T(S)$ is

$$
S \ominus T(S)=\{x \in X:\{x\} \oplus T(x) \subseteq S\},
$$

where $T(S)$ denotes the image of $S$ under the map $T$. Notice that, for a constant map $T$, the definition (6) is equivalent to the well-known Pontryagin difference. For the parametric Pontryagin difference of a convex set and a parametrically convex map we have the following result.

Lemma 2.3: Let $S \subseteq X$ be a convex set and let $T: X \rightarrow$ $\mathcal{P}(X)$ be a parametrically convex point-to-set map, then $S \ominus$ $T(S)$ is convex.

Proof: Define $Z=S \ominus T(S)$ and let $z_{1}, z_{2} \in Z$, then by definition of the parametric Pontryagin difference, we have

$$
\left\{z_{i}\right\} \oplus T\left(z_{i}\right) \subseteq S, i=1,2 .
$$

To see that $Z$ is convex we show that line segments between all possible $z_{1}$ and $z_{2}$ are subsets of $Z$, i.e. for all $\lambda \in[0,1]$,

$$
\begin{aligned}
\left\{\lambda z_{1}+(1-\lambda) z_{2}\right\} \oplus T\left(\lambda z_{1}+(1-\lambda) z_{2}\right) & \\
& \subseteq\left\{\lambda z_{1}+(1-\lambda) z_{2}\right\} \oplus \lambda T\left(z_{1}\right) \oplus(1-\lambda) T\left(z_{2}\right) \\
& \subseteq \lambda \underbrace{\left(\left\{z_{1}\right\} \oplus T\left(z_{1}\right)\right)}_{\subseteq S} \oplus(1-\lambda) \underbrace{\left(\left\{z_{2}\right\} \oplus T\left(z_{2}\right)\right)}_{\subseteq S} \subseteq S
\end{aligned}
$$

where the last inclusion follows from the convexity of $\mathcal{S}$.

Now consider a parametrically convex set of the form of (3).

Lemma 2.4: The point-to-set map $\mathcal{V}(x)$ defined by (3) is parametrically convex for all $x \in \mathcal{X} \subset \mathbb{R}^{n}$ if $H(x)$ is elementwise convex in $x \in \mathcal{X}$ (i.e. each element $H_{i}(x)$ of $H(x)$ satisfies $H_{i}\left(\lambda x_{1}+(1-\lambda) x_{2}\right) \leq \lambda H_{i}\left(x_{1}\right)+(1-\lambda) H_{i}\left(x_{2}\right)$ for all $\lambda \in[0,1]$ and $\left.x_{1}, x_{2} \in \mathcal{X}\right)$ and the representation $\{v: G v \leq H(x)\}$ is irredundant for all $x \in \mathcal{X}$.

Proof: To show that $\mathcal{V}\left(\lambda x_{1}+(1-\lambda) x_{2}\right) \subseteq \lambda \mathcal{V}\left(x_{1}\right) \oplus$ $(1-\lambda) \mathcal{V}\left(x_{2}\right)$ for all $\lambda \in[0,1]$ and $x_{1}, x_{2} \in \mathcal{X}$ we note that

$$
\begin{aligned}
& \mathcal{V}\left(\lambda x_{1}+(1-\lambda) x_{2}\right) \\
& =\left\{v: G v \leq H\left(\lambda x_{1}+(1-\lambda) x_{2}\right)\right\} \\
& \subseteq\left\{v: G v \leq \lambda H\left(x_{1}\right)+(1-\lambda) H\left(x_{2}\right)\right\} \\
& =\left\{v: G v \leq \lambda H\left(x_{1}\right)\right\} \oplus\left\{v: G v \leq(1-\lambda) H\left(x_{2}\right)\right\} \\
& =\lambda \mathcal{V}\left(x_{1}\right) \oplus(1-\lambda) \mathcal{V}\left(x_{2}\right) .
\end{aligned}
$$

Lemmas 2.3 and 2.4 imply that, if $\mathcal{V}(x)$ is defined by (3) in terms of an elementwise convex function $H(x)$, then the parametric Pontryagin difference $\mathcal{X} \ominus \mathcal{V}(\mathcal{X})$ is a convex set. As we show in the next section, if $\mathcal{X}$ is polytopic and $\mathcal{V}(x)$ is pointwise polytopic, then $\mathcal{X} \ominus \mathcal{V}(\mathcal{X})$ is also a polytope.

\section{Maximal Robust Positively InVariant Sets For State Dependent Disturbance}

In this section we describe an iterative algorithm to compute the MRPI set (2) for a linear system (1) subject to disturbances $v \in \mathcal{V}(x)$ where $\mathcal{V}(x)$ is a parametrically convex point-to-set map defined by (3) in terms of a convex piecewise affine function $H(x)$. We assume (without loss of generality) that the set $\mathcal{V}(x)$ is pointwise compact and polytopic for finite $x \in \mathcal{X}_{0}$ and can hence be represented as the convex hull of its vertices $\mathcal{V}(x)=\operatorname{conv}\left\{v_{i}(x)\right\}$. Since $\mathcal{V}(x)$ has a piecewise affine dependence on $x$ the vertices $v_{i}(x)$ are also piecewise affine in $x$. The set $\mathcal{X}^{\infty}$ as defined in (2) is required to satisfy $\Psi x+v \in \mathcal{X}^{\infty}$ for all $x \in \mathcal{X}^{\infty}$ and $v \in \mathcal{V}(x)$. To compute the MRPI set we start from the given state constraint set

$$
\mathcal{X}_{0}=\left\{x: \Xi_{0, i} x \leq \xi_{0, i} \forall i \in \mathcal{I}_{0}\right\}
$$

and recursively cut off points that cannot satisfy the invariance condition (2) by iteratively introducing constraints that exclude all points for which the successor state can lie outside $\mathcal{X}_{0}$. The first iteration enforces the constraint:

$$
\begin{aligned}
& \Xi_{0, i}(\Psi x+v) \stackrel{!}{\leq} \xi_{0, i} \forall v \in \operatorname{conv}\left\{v_{i}(x)\right\} \\
& \Xi_{0, i} \Psi x+\max _{v \in \mathcal{V}(x)} \Xi_{0, i} v \leq \xi_{0, i} \\
& \Xi_{0, i} \Psi x+\underbrace{\max _{j} \Xi_{0, i} v_{j}(x)}_{=v_{0, i}^{*}(x)} \leq \xi_{0, i} .
\end{aligned}
$$

for each $i \in \mathcal{I}_{0}$. Here $v_{0, i}^{*}(x)$ is not necessarily given by a unique maximiser, but is the solution of a multi-parametric linear program and hence is given by a vertex $v_{i}(x)$ of $\mathcal{V}(x)$ for each $x$ on that facet. Since each vertex is a piece-wise affine function of $x$, the maximum $v_{0, i}^{*}(x)$ is also piece-wise affine so the set $\mathcal{X}_{1}=\mathcal{X}_{0} \cap\left\{x: \Xi_{0, i} \Psi x+v_{0, i}^{*}(x) \leq \xi_{0, i} \forall i \in\right.$ $\left.\mathcal{I}_{0}\right\}$ has representation $\mathcal{X}_{1}=\left\{x: \Xi_{1, i} x \leq \xi_{1, i} \forall i \in \mathcal{I}_{1}\right\}$. The next iterate is defined by

$$
\begin{aligned}
\mathcal{X}_{2} & =\mathcal{X}_{1} \cap \\
& \left\{x: \Xi_{0, i} \Psi(\Psi x+v)+v_{0, i}^{*}(x) \leq \xi_{0, i} \forall i \in \mathcal{I}_{0}, v \in \mathcal{V}(x)\right\} \\
& =\mathcal{X}_{1} \cap\left\{x: \Xi_{0, i} \Psi^{2} x+v_{1, i}^{*}(x)+v_{0, i}^{*}(x) \leq \xi_{0, i} \forall i \in \mathcal{I}_{0}\right\}
\end{aligned}
$$

and at the $(k+1)$ st iteration we have

$$
\mathcal{X}_{k+1}=\mathcal{X}_{k} \cap\left\{x: \Xi_{0, i} \Psi^{k} x+\sum_{l=1}^{k-1} v_{l, i}^{*}(x) \leq \xi_{0, i} \forall i \in \mathcal{I}_{0}\right\},
$$

where

$$
\begin{aligned}
v_{l, i}^{*}(x)=\max _{j} \Xi_{0, i} \Psi^{l-1} v_{j}(x)=\max _{\tilde{v}} & \Xi_{0, i} \tilde{v} \\
\text { s.t. } & \tilde{v} \in \Psi^{l-1} \mathcal{V}(x)
\end{aligned}
$$

Remark 3.1: Determining the map $v_{l, i}(x)$ for all admissible $x \in \mathcal{X}_{0}$ in general poses a great classification challenge. Notice that in our calculations we can avoid determining the particular vertex $v_{l, i}(x)$ explicitly, it suffices to determine the vertex determining a supporting hyperplane of $\mathcal{X}_{k+1}$, i.e. $v_{l, i}^{*}(x)$. This can be done by adding all possible inequalities and applying a inequality reduction algorithm. In closed form the iterates can be expressed as

$$
\begin{aligned}
\mathcal{X}_{k+1} & =\mathcal{X}_{k} \cap\left(\Psi^{-1} \mathcal{X}_{k} \ominus \Psi^{k-1} \mathcal{V}\left(\mathcal{X}_{k}\right)\right)=\mathcal{X}_{k} \cap D_{k} \\
& =\bigcap_{0 \leq l \leq k+1}\left(\Psi^{-l} \mathcal{X}_{0} \bigodot_{1 \leq i \leq l-1} \Psi^{i} \mathcal{V}\left(\mathcal{X}_{l-1}\right)\right) .
\end{aligned}
$$


Notice that we do not require $\Psi$ to be invertible, in (7) $\Psi^{-1} \mathcal{X}_{k}$ merely denotes the preimage of $\mathcal{X}_{k}$ under the linear map $\Psi$. We will use (7) to prove the finite determinability of $\mathcal{X}^{\infty}$, namely that there exists a finite number $N$ such that $x \in \mathcal{X}_{N}$ implies $\Psi x+v \in \mathcal{X}_{N}$ for all $v \in \mathcal{V}(x)$ and hence $\mathcal{X}_{N}$ is robustly positively invariant.

Lemma 3.2: Let the state constraint set $\mathcal{X}_{0}$ be contained in a band: $\mathcal{X}_{0} \subseteq B=\{x: \Gamma x \leq \mathbf{1} \wedge-\Gamma x \leq 1\}$, let the pair $(\Psi, \Gamma)$ be observable and let $\mathcal{V}(x)$ be defined by (3) in terms of a piecewise affine function $H(x)$. Then $\mathcal{X}_{N} \subseteq \mathcal{X}_{N+1}$ for a finite $N$, and hence the MRPI set $\mathcal{X}^{\infty}=\mathcal{X}_{N}$ is a polytope.

Proof: From (7) it is clear that $\mathcal{X}^{\infty}=\emptyset$ if $\mathcal{X}_{k}=\emptyset$ for any $k \geq 0$. For the remainder of the proof we assume that the MRPI set $\mathcal{X}^{\infty}$ is non-empty. For this we require that $\bigcup_{s \in \mathcal{S}} \mathcal{V}(s)$ is bounded on any bounded set $\mathcal{S} \subset \mathbb{R}^{n}$.

The proof has two main steps: First we prove that $\mathcal{X}_{p}$ is bounded for $p \leq n$ where $n$ is the dimension of the state $x$. The second step is to prove that in (7) the set $D_{k}$ grows exponentially, i.e. that for any given bounded set $\mathcal{C}$ there exists finite $N$ such that $\mathcal{C} \subseteq D_{N}$. The proof is concluded by setting $\mathcal{C}=\mathcal{X}_{N}$ and deducing that $\mathcal{X}^{\infty}=\mathcal{X}_{N}$.

For the first step, notice that observability of $(\Psi, \Gamma)$ is equivalent to the observability matrix $\Omega$ having full rank, i.e. $\operatorname{rank}(\Omega)=\operatorname{rank}\left(\left[\Gamma^{T} \cdots\left(\Gamma \Psi^{n-1}\right)^{T}\right]\right)=n$. But this implies that the set

$$
\mathcal{P}_{n}=\{x: \Omega x \leq \mathbf{1} \wedge-\Omega x \leq \mathbf{1}\}=\bigcap_{0 \leq l \leq n-1} \Psi^{-l} B
$$

is bounded, and since $\mathcal{X}_{k} \subseteq \mathcal{P}_{k}$ for all $k$, the set $\mathcal{X}_{n}$ is also bounded. To show that $D_{k}$ grows exponentially we use the boundedness of $\mathcal{V}\left(\mathcal{X}_{n}\right)$. Let $K_{1}$ denote the smallest ball that contains $\mathcal{V}\left(\mathcal{X}_{n}\right)$ and let $\rho$ denote the spectral radius of $\Psi$. Furthermore, let $K_{2}$ be the biggest ball that is contained in $\mathcal{X}_{0}$. From (7), $D_{k}$ has the representation

$$
D_{k}=\underbrace{\Psi^{-k} \mathcal{X}_{0}}_{=\mathcal{S}_{1}} \ominus \underbrace{\left(\bigoplus_{1 \leq i \leq k-1} \Psi^{i} \mathcal{V}\left(\mathcal{X}_{k}\right)\right)}_{=\mathcal{S}_{2}} .
$$

Since $\Psi$ is asymptotically stable we have $\rho<1$ and therefore $\rho^{-k} K_{2} \subseteq \Psi^{-k} K_{2} \subseteq \Psi^{-k} \mathcal{X}_{0}=\mathcal{S}_{1}$, implying exponential growth of $D_{k}$ provided $\mathcal{S}_{2}$ is bounded. To bound $\mathcal{S}_{2}$ we note that for $k \geq n$ we have $\mathcal{S}_{2} \subseteq \bigoplus_{1 \leq i \leq k-1} \rho^{i} K_{1} \subseteq \frac{1}{1-\rho} K_{1}$. Thus $D_{k}$ grows exponentially, whereas $\mathcal{X}_{k}$ is contained in a ball of finite radius, and we conclude from (7) that $\mathcal{X}_{k+1}=$ $\mathcal{X}_{k}$ for all $k \geq N$ and hence $\mathcal{X}_{N}=\mathcal{X}^{\infty}$ for finite $N$.

We have seen that we can compute the maximal robust positively invariant set $\mathcal{X}^{\infty}$ for linear systems with state dependent constraints. In the next section we will illustrate the concept with an example.

\section{EXAMPLE}

This section discusses the calculation of the MRPI set for a linearised, simplified model of the magnetic levitation system depicted in figure 1. The system dynamics for the ball are given by $m \ddot{y}=m g-c \frac{i^{2}}{y^{2}}$, where $m, g, c, i$ and $y$ denote the mass of the ball, the gravitational constant, a constant factor,

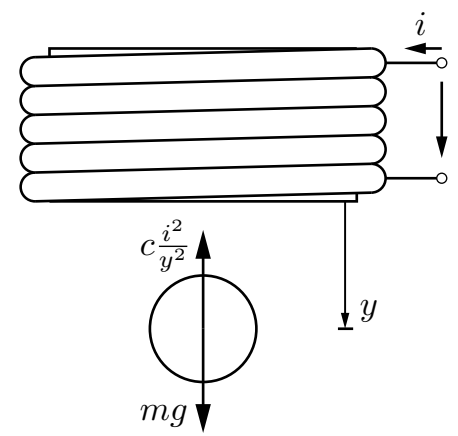

Fig. 1. Levitating ball system.

the current and the distance between the coil and the centre of the ball respectively. For illustration purposes we neglect inductive dynamics, using the current $u=i$ as the control input and the position $y$ and its first derivative $\dot{y}$ as the states: $x=(y, \dot{y})^{T}$. We find that any equilibrium has $\dot{y}=0$ and $u=\sqrt{\frac{g m}{c}} y$ for any positive position $y>0$. Linearising the nonlinear differential equation $\dot{x}=f(x, u)$ around an equilibrium point $(\hat{x}, \hat{u})$ gives the approximate linear model

$$
\Delta \dot{x}=\underbrace{\left(\begin{array}{cc}
0 & 1 \\
\frac{c \hat{x}^{2}}{m \hat{x}_{1}^{3}} & 0
\end{array}\right)}_{\frac{\partial f}{\partial x}(\hat{x}, \hat{u})} \Delta x+\underbrace{\left(\begin{array}{c}
0 \\
-\frac{2 c \hat{u}}{m \hat{x}_{1}^{2}}
\end{array}\right)}_{\frac{\partial f}{\partial u}(\hat{x}, \hat{u})} \Delta u
$$

where $\Delta u=u-\hat{u}$ and $\Delta x \approx x-\hat{x}$. We derive the discrete time dynamics with sampling rate $T_{s}$ using the Euler formula $x^{+}=x+T_{s} f(x, u)=: \tilde{f}(x, u)$ giving $\Delta x^{+}=A \Delta x+B \Delta u$,

$$
A=I+T_{s} \frac{\partial f}{\partial x}(\hat{x}, \hat{u}), \quad B=T_{s} \frac{\partial f}{\partial u}(\hat{x}, \hat{u})
$$

Although this system has a control input, the algorithm for computing the MRPI set is applicable since we consider the closed loop system under linear feedback $u=K x$, where $K$ satisfies the robust Lyapunov condition $V(x)-V((A+$ $B K) x+v) \leq \gamma^{2} v^{T} v$ with $V(x)=x^{T} P x \geq 0$, i.e. $x^{T} P x-$ $((A+B K) x+v)^{T} P((A+B K) x+v) \geq x^{T}\left(Q+K^{T} R K\right) x-$ $\gamma^{2} v^{T} v$ for a minimum $\gamma^{2}$, see e.g. [13]. A representation of additive disturbances acting on the linearised model due to linearisation errors, i.e. a system representation $x^{+}=A x+$ $B u+v$ with an additive disturbance $v$, can be obtained using the Mean Value Theorem (e.g. [14]).

Lemma 4.1 (Mean Value Theorem): Let $g: \mathcal{X} \rightarrow \mathbb{R}^{m}$ be continuously differentiable, $\mathcal{X} \subset \mathbb{R}^{n}$ be open, and $x \in \mathcal{X}$, $h \in \mathbb{R}^{n}$ be such that $x+t h \in \mathcal{X}$ for all $t \in[0,1]$. Then

$$
g(x+h)=g(x)+\left(\int_{0}^{1} \frac{\partial g}{\partial x}(x+t h) d t\right) \cdot h .
$$

Using the Mean Value Theorem and the linearisation around $(\hat{x}, \hat{u})$, and defining $\tilde{x}=x-\hat{x}, \tilde{u}=u-\hat{u}$, we obtain the successor state $x^{+}=\tilde{f}(\hat{x}+\tilde{x}, \hat{u}+\tilde{u})$ as

$$
\begin{aligned}
x^{+}=\tilde{f}(\hat{x}, \hat{u})+\int_{0}^{1} \frac{\partial \tilde{f}}{\partial x} & (\hat{x}+t \tilde{x}, \hat{u}+t \tilde{u}) d t \cdot \tilde{x} \\
& +\int_{0}^{1} \frac{\partial \tilde{f}}{\partial u}(\hat{x}+t \tilde{x}, \hat{u}+t \tilde{u}) d t \cdot \tilde{u}
\end{aligned}
$$

and hence 


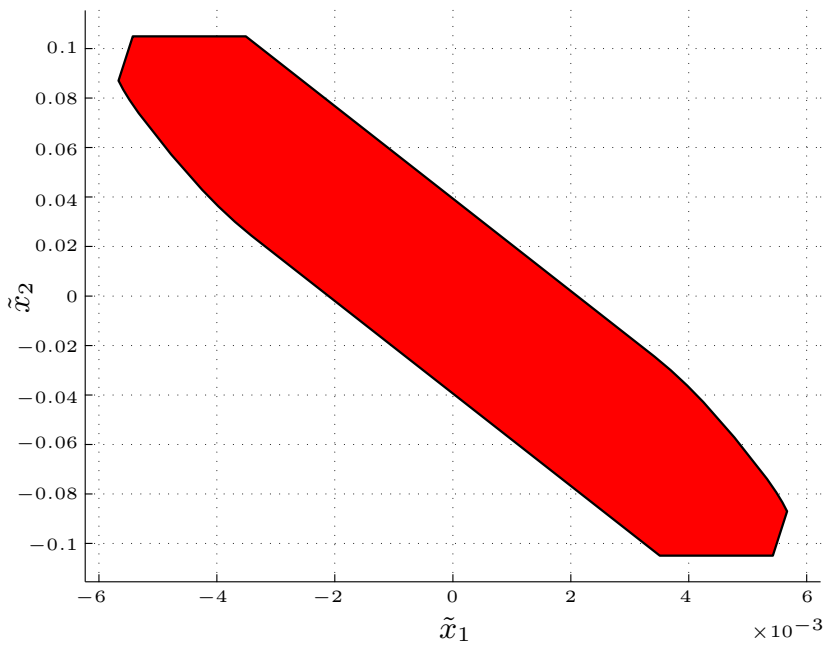

Fig. 2. The maximal robust positively invariant set for the levitating ball.

$$
\begin{aligned}
\tilde{x}^{+}=A \tilde{x}+B \tilde{u} & +\left(\int_{0}^{1} \frac{\partial \tilde{f}}{\partial x}(\hat{x}+t \tilde{x}, \hat{u}+t \tilde{u}) d t-A\right) \tilde{x} \\
& +\left(\int_{0}^{1} \frac{\partial \tilde{f}}{\partial u}(\hat{x}+t \tilde{x}, \hat{u}+t \tilde{u}) d t-B\right) \tilde{u}
\end{aligned}
$$

This implies the dynamics

$$
\tilde{x}^{+}=A \tilde{x}+B \tilde{u}+H^{x} \tilde{x}+H^{u} \tilde{u}
$$

where $H^{x}$ and $H^{u}$ can be determined by integration. If $f(x, u)$ is continuously differentiable for all $x \in \mathcal{X}$ and $u \in \mathcal{U}$, where $\mathcal{X}, \mathcal{U}$ are compact sets, then we can bound the values of $H^{x} \tilde{x}+H^{u} \tilde{u}$ by a finite convex combination of extremal values, i.e. $H^{x} \tilde{x}+H^{u} \tilde{u} \in \operatorname{conv}_{k}\left\{H_{k}^{x} \tilde{x}+H_{k}^{u} \tilde{u}\right\}$. Thus we can introduce the elementwise disturbance bound

$$
\begin{aligned}
\mathcal{V}(\tilde{x}, \tilde{u}) & =\left\{v: \min _{k}\left\{H_{k, i}^{x} \tilde{x}+H_{k, i}^{u} \tilde{u}\right\} \leq v_{i} \wedge\right. \\
v_{i} & \left.\leq \max _{k}\left\{H_{k, i}^{x} \tilde{x}+H_{k, i}^{u} \tilde{u}\right\}, i=1, \ldots, n\right\} .
\end{aligned}
$$

Therefore $\tilde{x}^{+}=A \tilde{x}+B \tilde{u}+v$ accounts for all nonlinearities within $\mathcal{X} \times \mathcal{U}$ if $v \in \mathcal{V}(\tilde{x}, \tilde{u})$. For general nonlinear systems finding the extremal values of $\left(H^{x}, H^{u}\right)$ is non-trivial. To obtain values for $\left(H_{k}^{x}, H_{k}^{u}\right)$ we sample $\mathcal{X} \times \mathcal{U}$ and evaluate the integral expressions defining $\left(H_{k}^{x}, H_{k}^{u}\right)$ pointwise. This leads to an inner approximation of the linearisation error set, however we can make it as tight as necessary by increasing the number of samples. For this we use the numerical values for the example of the levitating ball: $T_{s}=30 \mathrm{~ms}, C=1$, $m=100 \mathrm{~g}, \hat{x}_{1}=50 \mathrm{~mm}$ and $\mathcal{X}=\left\{x:\left|x_{1}-\hat{x}_{1}\right| \leq\right.$ $\left.1 \mathrm{~mm} \wedge\left|x_{2}\right| \leq 105 \mathrm{~mm} / \mathrm{s}\right\}, \mathcal{U}=\{u:|u-\hat{u}| \leq 10 \mathrm{~mA}\}$. Using a total of 25 samples for the computation of $\left(H^{x}, H^{u}\right)$ we obtain the invariant set shown in figure 2 , which is less conservative than using fixed bounds on the nonlinearities (this will be shown in Section VI). The algorithm for computing the MRPI set terminates after 3 iterations.

\section{Maximal Robust Positively InVARiant Sets for PARAMETRISED DISTURBANCE}

In this section we describe the computation of the MRPI set for (1) with a disturbance set parametrised by a scalar $\theta$ :

$$
\mathcal{V}(\theta)=\{v: G v \leq(1+\theta) \mathbf{1}\}=(1+\theta) \mathcal{V}(0), \quad \theta>-1 .
$$

For input constrained systems under a given feedback law, we combine the uniform scaling of the disturbance set $\mathcal{V}(\theta)$ with non-uniform scaling of the input constraint set

$$
\mathcal{U}(\alpha)=\left\{u: F u \leq\left(I+\operatorname{diag}\left(\alpha_{1}, \ldots, \alpha_{p}\right)\right) \mathbf{1}\right\} .
$$

The necessity of uniform scaling of the disturbance constraints is due to the method we use to avoid solving multiparametric linear programs in every step of the proposed iteration. A representation of the MRPI set of a system parametrised with respect to a scaling parameter allows us to study the system's sensitivity to stronger/weaker disturbances; similarly analysing the sensitivity to scaling of input constraints can be useful to choose particular actuators.

Remark 5.1: The set $\mathcal{V}(\theta)$ is non-empty and contains the origin for all $\theta>-1$ and hence the maximum

$$
\begin{aligned}
0<\max _{v} & c^{T} v \\
\text { s.t. } & G v \leq(1+\theta) \mathbf{1}
\end{aligned}
$$

is positive for any non-zero $c$.

In the following we describe an algorithm to compute the MRPI set $\mathcal{Z}^{\infty}$ contained in $\mathcal{Z}=\left\{(x, \theta): \mathcal{F}_{i} x+\mathcal{G}_{i} \theta \leq\right.$ $1, \forall i \in \mathcal{I}\}$. As in the state dependent case we iteratively introduce constraints separating points for which the successor state can lie outside the previous set, i.e. starting from $Z_{0}=\mathcal{Z}$ we determine the first iterate by enforcing all individual constraints onto all possible successor states: $Z_{1}=Z_{0} \cap D_{0}$ where $D_{0}$ is defined by

$$
\begin{aligned}
& D_{0}=\left\{\mathcal{F}_{i}(\Psi x+v)+\mathcal{G}_{i} \theta \leq 1 \forall v \in \mathcal{V}(\theta), i \in \mathcal{I}_{0}\right\} \\
& =\left\{\begin{array}{cl}
\mathcal{F}_{i} \Psi x+\max _{v} & F_{i} v \\
\text { s.t. } & G v \leq(1+\theta) \mathbf{1}
\end{array}+\mathcal{G}_{i} \theta \leq 1 \forall i \in \mathcal{I}_{0}\right\} \\
& =\{\mathcal{F}_{i} \Psi x+(1+\theta) \underbrace{\max _{v} F_{i} v \quad G v \leq \mathbf{1}}_{v_{0, i}^{*}} \text { s.t. } G v \mathcal{G}_{i} \theta \leq 1 \forall i \in \mathcal{I}_{0}\} \\
& =\left\{\mathcal{F}_{i} \Psi x+\left(\mathcal{G}_{i}+v_{0, i}^{*}\right) \theta \leq 1-v_{0,1}^{*} \forall i \in \mathcal{I}_{0}\right\}
\end{aligned}
$$

Using the same principle we define $Z_{k+1}=Z_{k} \cap D_{k}$ with $D_{k}$ given by

$D_{k}=\left\{\mathcal{F}_{i} \Psi^{k+1} x+\left(\mathcal{G}_{i}+\sum_{0 \leq l \leq k} v_{l, i}^{*}\right) \theta \leq 1-\sum_{0 \leq l \leq k} v_{l, i}^{*} i \in \mathcal{I}_{0}\right\}$

where we use

$$
\begin{aligned}
v_{l, i}^{*}=\max _{v} & \mathcal{F}_{i} \Psi^{l} v \\
\text { s.t. } & G v \leq \mathbf{1}
\end{aligned}
$$

Notice that (14) can be represented in various ways:

$$
\begin{aligned}
& \max _{v} \mathcal{F}_{i} \Psi^{l} v=\max _{\tilde{v}} \mathcal{F}_{i} \tilde{v}=\max _{\tilde{v}} \mathcal{F}_{i} \tilde{v}
\end{aligned}
$$

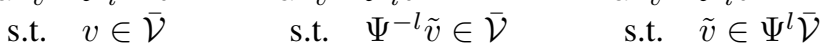


where $\overline{\mathcal{V}}=\mathcal{V}(0)$ for notational convenience. For any fixed $\hat{\theta}>-1$ we have the closed form description

$$
\begin{aligned}
\left.Z_{k+1}\right|_{\hat{\theta}} & =\left.Z_{k}\right|_{\hat{\theta}} \cap\left(\left.\Psi^{-1} Z_{k}\right|_{\hat{\theta}} \ominus \Psi^{k-1} \mathcal{V}(\hat{\theta})\right) \\
& =\bigcap_{0 \leq l \leq k}\left(\left.\Psi^{-l} \mathcal{Z}\right|_{\hat{\theta}} \bigodot_{0 \leq i \leq l-1} \Psi^{i} \mathcal{V}(\hat{\theta})\right) .
\end{aligned}
$$

The iteration terminates when $Z_{k} \subseteq Z_{k+1}$. As in section III we will require $\left.\mathcal{Z}\right|_{\hat{\theta}}$ to be contained in an observable band: $\left.\mathcal{Z}\right|_{\hat{\theta}}=\{x: \mathcal{F} x \leq \mathbf{1}-\mathcal{G} \hat{\theta}\} \subseteq \mathcal{B}=\{x: \Gamma x \leq \mathbf{1} \wedge-\Gamma x \leq \mathbf{1}\}$ for all $\hat{\theta}>-1$. We have the following result:

Lemma 5.2: Let the system constraints be contained in a band $\left.\mathcal{Z}\right|_{\hat{\theta}} \subseteq \mathcal{B}=\{x: \Gamma x \leq \mathbf{1} \wedge-\Gamma x \leq \mathbf{1}\}$ for any fixed $\hat{\theta}$, let the pair $(\Psi, \Gamma)$ be observable and let $\mathcal{V}(0)$ be bounded, then $Z_{N} \subseteq Z_{N+1}$ for a finite $N$. Hence the MRPI set $\mathcal{Z}^{\infty}=Z_{N}$ is a finite polyhedron.

Proof: The proof is similar to that of Lemma 3.2. First we argue that for each fixed $\hat{\theta}$ the set $\left.Z_{k}\right|_{\hat{\theta}}$ becomes compact using the same observability argument as in Lemma 3.2. We then use the representation (15) to argue that $D_{k}$ in (13) grows exponentially and hence contains any compact set after a finite number of iterations. Fixing $\theta=\hat{\theta}$ does not affect the result since our argument is constructed for a fixed matrix $\Gamma$ with rows scaled by $\left(1-\mathcal{G}_{i} \hat{\theta}\right)^{-1}$; clearly we can re-scale the rows to accommodate any other choice of $\hat{\theta}>-1$.

The iterative process described above allows uniform scaling of the disturbance set. As mentioned earlier, the algorithm can be extended to the case of non-uniformly scaled input constraints to accommodate more degrees of freedom for system analysis simply by using

$$
\mathcal{Z}=\{(x, \theta, \alpha): \mathcal{F} x+\mathcal{G} \theta+\mathcal{H} \alpha \leq \mathbf{1}\} .
$$

This does not affect the algorithm since at each step of the iteration the elements of $\mathcal{H}$ remain unchanged.

\section{EXAMPLES}

In this section we compute MRPI sets for the levitating ball system presented in section IV and for a purely numerical model. These examples illustrate the effectiveness of the proposed approach for state dependent disturbances and the use of parametrised MRPI sets as a system analysis tool.

First we present the parametrised MRPI set for the levitating ball. To obtain results comparable to those in Section IV we derive fixed bounds on the effect of nonlinearities on each state by finding a set $\mathcal{V}$ that contains $\mathcal{V}(x, u)$ in (11) for all $(x, u) \in \mathcal{X} \times \mathcal{U}$. We also introduce a scaling parameter $\alpha$ such that $\mathcal{U}(0)=\mathcal{U}$ for $\alpha=0$. The MRPI set then has the form $\mathcal{Z}^{\infty}=\left\{(x, \theta, \alpha): \Lambda_{i}^{x} x+\Lambda_{i}^{\theta} \theta+\Lambda_{i}^{\alpha} \alpha \leq \lambda_{i} \forall i \leq m_{\infty}\right\}$. Since $\alpha$ was introduced as a scaling parameter for $\mathcal{U}(\alpha)=$ $\{u: F u \leq(1+\alpha) \mathbf{1}\}$, all entries in $\mathcal{H}$ are non positive and remain unchanged throughout the computation of $\mathcal{Z}^{\infty}$; hence $\Lambda^{\alpha}$ has only non positive entries, and increasing $\alpha$ enlarges the parametrised MRPI set $\left.\left.\mathcal{Z}^{\infty}\right|_{\alpha_{1}} \subseteq \mathcal{Z}^{\infty}\right|_{\alpha_{2}}$ for $\alpha_{1} \leq \alpha_{2}$.

To compare the MRPI set $\mathcal{X}^{\infty}$ for state dependent disturbance constraints with the parametrised set $\mathcal{Z}^{\infty}$, we determine $\alpha_{\text {min }}$ such that $\left.\mathcal{X}^{\infty} \subseteq \mathcal{Z}^{\infty}\right|_{\left(\theta=0, \alpha=\alpha_{\min }\right)}$ by solving

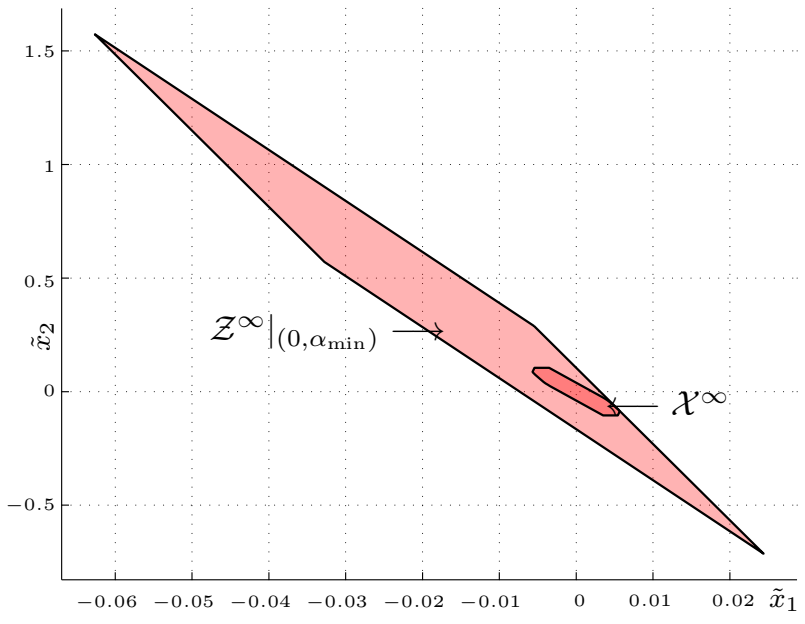

Fig. 3. MRPI sets for the minimally scaled fixed disturbance set and for the piecewise affine disturbance set. The MRPI set for fixed disturbance bounds is non-symmetric because the nonlinearities of the system dynamics are not symmetrical about the equilibrium.

$m_{\infty}$ linear programs:

$$
\begin{aligned}
\gamma_{i}^{*}=\max _{x} \Lambda_{i}^{x} x \\
\text { s.t. } x \in \mathcal{X}^{\infty}
\end{aligned}
$$

The minimal value for $\alpha_{\min }$ is then given by the maximal $\alpha$ satisfying $\gamma_{i}^{*}+\Lambda_{i}^{\theta} \cdot 0+\Lambda_{i}^{\alpha} \alpha \leq \lambda_{i}$. This gives the minimal value of $\alpha_{\min }=1.7555$ and the MRPI sets shown in Figure 3. The computation terminates after seven iterations and produces a polyhedron $\mathcal{Z}^{\infty}$ supported by $m_{\infty}=10$ planes. Caution is advised when interpreting $\alpha>0$, since the system is nonlinear, however $\alpha_{\min }>0$ indicates that the input constraint set $\mathcal{U}(0)=\mathcal{U}$ is too small to cope with a fixed disturbance set bounding the linearisation errors.

In the second example, we compute the parametrised MRPI set for the system

$$
x^{+}=\underbrace{\left(\begin{array}{ll}
1 & 2 \\
0 & 1
\end{array}\right)}_{A} x+\underbrace{\left(\begin{array}{cc}
1 & 1 \\
1 & -1
\end{array}\right)}_{B} u+\underbrace{\left(\begin{array}{ll}
1 & 2 \\
2 & 1
\end{array}\right)}_{D} w .
$$

We use the constraint sets $\mathcal{U}(\alpha)=\{u: F u \leq(I+$ $\operatorname{diag}(\alpha)) \mathbf{1} \wedge-F u \leq(I+\operatorname{diag}(\alpha)) \mathbf{1}\}$ and $\mathcal{V}(\theta)=D \mathcal{W}(\theta)$ where $\mathcal{W}(\theta)=\left\{w:\left|w_{1}\right| \leq 0.1(1+\theta) \wedge\left|w_{2}\right| \leq 0.15(1+\theta)\right\}$. The constraint matrix $F$ is given by

$$
F=\left(\begin{array}{cc}
2 & 2 \\
4 & 0 \\
2 & -2
\end{array}\right)
$$

The constraints of this example were chosen for illustrative purposes. We now analyse the effects of changing individual constraints on the control input (by changing $\alpha)$ as well as the effect of scaling the perturbations $w$ (through $\theta$ ). To initialise the iteration we use $\mathcal{Z}(x, \theta, \alpha)=$ $\left\{(x, \theta, \alpha): K x \in \mathcal{U}(\alpha) \wedge K_{w} x \in \mathcal{W}(\theta) \wedge \alpha, \theta>\mathbf{- 1}\right\}$, for $K$ and $K_{w}$ satisfying the robust Lyapunov condition $x^{T} P x-$ $((A+B K) x+D w)^{T} P((A+B K) x+D w) \geq x^{T}(Q+$ $\left.K^{T} R K\right) x-\gamma^{2} w^{T} w$, i.e. $K_{w}=\left(\gamma^{2}-D^{T} P D\right) D^{T} P(A+$ $B K)$ for $Q=\operatorname{diag}(2,1)$ and $R=I$. Using these numerical 


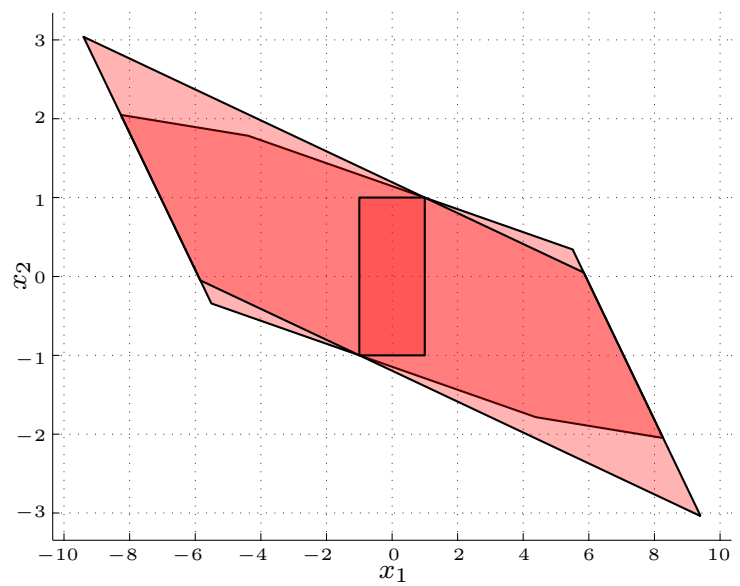

Fig. 4. The extremal MRPI sets $\left.\mathcal{Z}^{\infty}\right|_{\left(\theta_{\min }, 0\right)}$ and $\left.\mathcal{Z}^{\infty}\right|_{\left(\theta_{\max }, 0\right)}$ containing the unit box.

values we determine the MRPI set $\mathcal{Z}^{\infty}$. After 22 iterations the algorithm terminates producing a total of 61 facets. By examining the conditions on $\mathcal{H}$ and the scaling parameters $\alpha$, it is possible to show that $\Lambda^{\alpha}$ can have at most one nonzero entry per row. We can therefore compute which $\alpha_{i}$ will change the MRPI set the most. This can be done elementwise by calculating

$$
\max _{i} \frac{-\Lambda_{i}^{\alpha}}{\left\|\Lambda_{i}^{x}\right\|_{2}} .
$$

For this example the greatest sensitivity corresponds to $\alpha_{3}$, i.e. the third input constraint. As for the first example we might also want to know how much disturbance the closed loop system can tolerate such that a given set is contained in the MRPI set $\left.\mathcal{C} \subseteq \mathcal{Z}^{\infty}\right|_{(\theta, 0)}$. Although non-positivity of $\mathcal{H}$ implies non-positivity of $\Lambda^{\alpha}$, a similar argument cannot be applied to $\Lambda^{\theta}$. Let $\left(\Lambda^{1}, \lambda^{1}\right)$ and $\left(\Lambda^{2}, \lambda^{2}\right)$ denote all rows of $\Lambda^{\theta}$ such that $\Lambda^{1, \theta}>0$ and $\Lambda^{2, \theta} \leq 0$. This implies that for any fixed $\hat{\alpha}$ the set $\left.\mathcal{Z}^{\infty}\right|_{\hat{\alpha}}$ is compact, and since $\left.\mathcal{Z}^{\infty}\right|_{(\hat{\theta}, \hat{\alpha})}$ is compact, there exist a maximal $\theta_{\max }$ and likewise a minimal $\theta_{\text {min }}$ such that $\Lambda^{1, x} x+\Lambda^{1, \alpha} \hat{\alpha}+\Lambda^{1, \theta} \theta \leq \lambda^{1}$ can be satisfied. We can solve the optimisation programs

$$
\begin{aligned}
\gamma_{i}=\max _{x} & \Lambda_{i}^{1, x} x \\
\text { s.t. } & x \in \mathcal{C}
\end{aligned} \quad \delta_{i}=\max _{x} \Lambda_{i}^{2, x} x .
$$

The extremal values for which $\mathcal{C}$ is contained in the MRPI set are given by the smallest $\theta_{\max }$ satisfying $\gamma_{i}+\Lambda_{i}^{1, \alpha} \hat{\alpha}+$ $\Lambda_{i}^{1, \theta} \theta_{\max } \leq \lambda_{i}^{1}$ and the largest $\theta_{\min }$ satisfying $\delta_{i}+\Lambda_{i}^{2, \alpha} \hat{\alpha}+$ $\Lambda_{i}^{2, \theta} \theta_{\min } \leq \lambda_{i}^{2}$ The numerical values for the example are given by $\left[\theta_{\min }, \theta_{\max }\right]=[-0.9999,6.2582]$. Two- and threedimensional illustrations of the parametrised MRPI set are given in Figure 4 and 5 respectively.

\section{CONCLUSIONS}

This paper discusses extensions to existing computational methods for determining MRPI sets for linear systems subject to additive disturbance for two descriptions of the sets bounding unknown additive disturbances. The case of state dependent disturbances was considered, and applied to the problem of determining approximations to MRPI sets

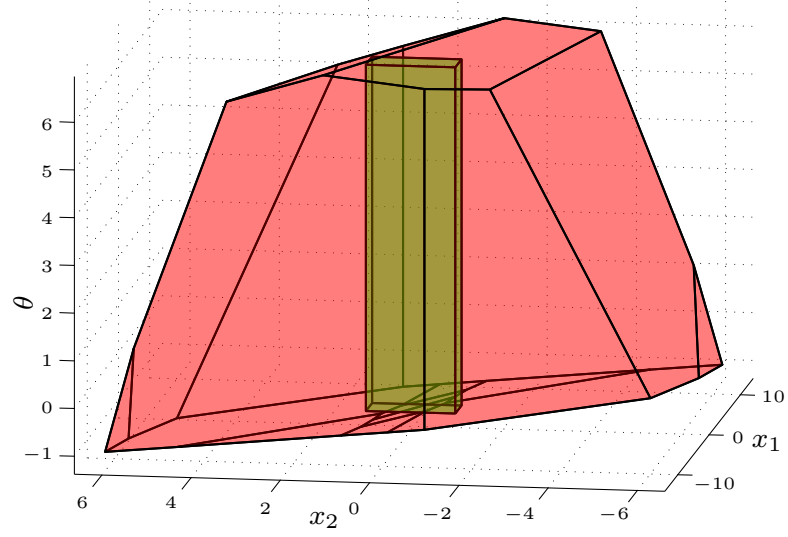

Fig. 5. The parametrised MRPI set for $\alpha=0$. Containing the unit box in the interior between $\theta_{\min }$ and $\theta_{\max }$.

for linearised nonlinear systems. This was illustrated using the example of a magnetic levitation system. Secondly the computation of MRPI sets for systems with disturbance and control inputs bounded by scaled sets was considered. This enables system analyses based on uniform scaling applied to disturbance sets and non-uniform scaling of input constraints. A comparison of the two approaches shows the effectiveness of the method based on state dependent disturbance sets.

\section{REFERENCES}

[1] F. Blanchini and S. Miani, Set-Theoretic Methods in Control. Springer, London, 2008.

[2] J. Glover and F. Schweppe, "Control of linear dynamic systems with set constrained disturbances," Automatic Control, IEEE Transactions on, vol. 16, no. 5, pp. 411-423, Oct 1971.

[3] D. Bertsekas and I. Rhodes, "On the minimax reachability of target sets and target tubes," Automatica, vol. 7, pp. 233-247, 1971.

[4] D. Mayne, J. Rawlings, C. Rao, and P. Scokaert, "Constrained model predictive control: Stability and optimality," Automatica, vol. 36, no. 6, pp. 789-814, 2000.

[5] F. Blanchini, "Ultimate boundedness control for uncertain discretetime systems via set-induced lyapunov functions," Automatic Control, IEEE Transactions on, vol. 39, no. 2, pp. 428-433, Feb 1994.

[6] E. De Santis, "On positively invariant sets for discrete-time linear systems with disturbance: an application of maximal disturbance sets," Automatic Control, IEEE Transactions on, vol. 39, no. 1, pp. 245-249, Jan 1994.

[7] I. Kolmanovsky and E. Gilbert, "Theory and computation of disturbance invariant sets for discrete-time linear systems." Mathematical Problems in Engineering, vol. 4, no. 4, pp. 317-367, 1998.

[8] F. Blanchini, "Feedback control for linear time-invariant systems with state and control bounds in the presence of disturbances," Automatic Control, IEEE Transactions on, vol. 35, no. 11, pp. 1231-1234, Nov 1990.

[9] — "Constrained control for uncertain linear systems," J. Optimization Theory and Applications, vol. 71, no. 3, pp. 465-484, 1991.

[10] V. Kuntsevich and B. Pshenichnyi, "Minimal invariant sets of dynamic systems with bounded disturbances," Cybernetics and Systems Analysis, vol. 32, no. 1, pp. 58-64, 1996.

[11] S. Raković, E. Kerrigan, D. Mayne, and J. Lygeros, "Reachability analysis of discrete-time systems with disturbances," IEEE Transactions on Automatic Control, vol. 51, no. 4, pp. 546-561, 2006.

[12] W. Hogan, "Point-to-set maps in mathematical programming," SIAM Review, vol. 15, no. 3, pp. 591-603, 1973.

[13] S. Boyd, L. El Ghaoui, E. Feron, and V. Balakrishnan, Linear Matrix Inequalities in System and Control Theory, ser. Studies in Applied Mathematics. Philadelphia, PA: SIAM, Jun. 1994, vol. 15.

[14] T. M. Apostol, Mathematical Analysis. Addison-Wesley, 1974. 